\title{
Harald Lutz zum 80. Geburtstag - ein Leben mit dem Ultraschall
}

Heute gilt es einem der profiliertesten Pioniere des Ultraschalls zum 80 . Geburtstag zu gratulieren und seine Leistungen für den Ultraschall zu würdigen.

Als Rettenmaier-Schüler wurde Harald Lutz kurz nach der Gründung Mitglied der DAUD (Deutsche Arbeitsgemeinschaft für Ultraschalldiagnostik) und war von Anfang aktiver Teilnehmer an deren Jahrestagungen. 1973 ernannte Prof. Dr. Demling ihn zum Leiter des renommierten Erlanger Ultraschalllabors und Nachfolger Rettenmaiers.

Seine ersten wissenschaftlichen Arbeiten erfolgten 1972/73 zusammen mit Rettenmaier zur Diagnostik von Lymphknoten und MagenDarm-Tumoren, die er mit dem prägenden Begriff „Kokarde“ beschrieb. Aus seinem Labor folgten in den folgenden Jahren zahlreiche klinische Arbeiten zur Nieren-, Leber-, Gallenstein- und Pankreasdiagnostik, sowie erste Publikationen zur ultraschall-gestützten Punktion.

Als einer der ersten erkannte er 1975 das Potenzial der Multiarrayscanner und nutzte konsequent diese neue Technik. Bereits 1974 gründete er die Ultraschallschule Erlangen, die einzige, die bis heute ohne Unterbrechung Ultraschallkurse in Deutschland veranstaltet.

Harald Lutz habilitierte sich 1978 über Pankreassonografie und im gleichen Jahr erreichte seine Monografie „Ultraschalldiagnostik (B-scan) in der Inneren Medizin“ die rasch wachsende Ultraschall-Gemeinde. Es war das sehnlichst erwartete erste deutschsprachige Ultraschallbuch: Auf 140 Seiten ließ sich damals die gesamte internistische Sonografie darstellen.

1983 verantwortete er als Kongresspräsident das 7. Dreiländertreffen in Erlangen.

Ins Jahr 1983 fielen für Harald Lutz zahlreiche lebensentscheidende Karriereschritte. So wurde er zum außerplanmäßigen Professor für Innere Medizin ernannt und zum Chefarzt der Medizinischen Klinik I am Klinikum Bayreuth gewählt, dessen Ärztlicher Direktor er später wurde.
Ebenfalls 1983 wurde er zum Präsidenten der DEGUM gewählt - ein Amt, das er geschickt und tatkräftig bis 1986 ausübte. Vermutlich weckten die bereits damals nicht einfachen und eher un(ter)entwickelten Beziehungen der DEGUM zur EFSUMB sein Interesse an internationalen Gremien. Durch seine stetige, solide und zuverlässige Mitarbeit, die frei von persönlichen Allüren war, erwarb er sich mit diplomatischem Geschick hohes internationales Ansehen und wurde schließlich zum Präsidenten der EFSUMB (1990 - 1993) und der Ultraschallweltförderation (WFUMB 1997 -2000) gewählt, in deren Gremien für Ultraschallausbildung und Ultraschall in der 3. Welt er noch heute aktiv mitarbeitet. 2001 wurde Harald Lutz Ehrenmitglied der DEGUM.

Große und bleibende Verdienste hat Harald Lutz sich mit der Herausgabe des mittlerweile in 2. Auflage erschienenen 5-bändigen WHO Manual of Diagnostic Ultrasound erworben.

Wir, die Verfasser, kennen Harald Lutz seit der Gründungszeit der DAUD beziehungsweise der DEGUM. Uns verbindet eine langjährige Freundschaft, und wir blicken mit Freude auf eine fruchtbare Zusammenarbeit mit ihm zurück.

Harald Lutz war und ist für Bernd FrentzelBeyme der Motor des Ultraschallmuseums seit der Gründung 1993. Seither hat Harald Lutz keine Sitzung versäumt, ohne ihn läuft und lief im Museum nichts. Er ist der, der die alten Geräte und ihre Technik am besten versteht und beherrscht. Innerhalb des Museums arbeitet er unermüdlich an zahlreichen Projekten - und auch für die einfachsten Arbeiten ist er sich nicht zu schade. Zweifelsohne widmet er der Museumsarbeit seine bevorzugte Aufmerksamkeit und ist wegen seiner Kenntnisse der Ultraschallgeschichte unersetzlich.

Svein Ödegaard hat beste Erinnerungen an seine erste Begegnung mit Harald Lutz 1978 in Stuttgart. Seine Vorlesung hat ihn damals als Neuling in der Ultraschallwelt begeistert und stimuliert. In den folgenden Jahren hat Harald Lutz für Svein Ödegaard und die norwegischen Ultraschalldiagnos-

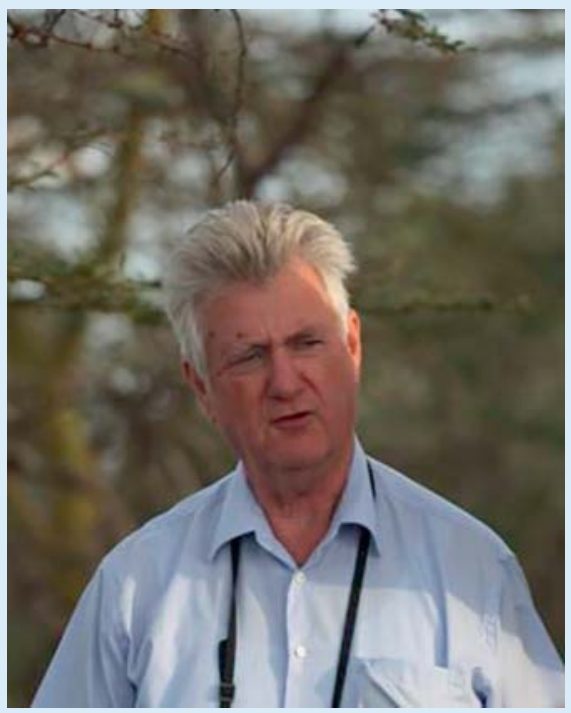

Harald Lutz

tiker viel bedeutet. Er war mehrmals in Norwegen und hat exzellente Vorlesungen gegeben, und als er die Zeitschrift „Ultraschall in Klinik und Praxis" gründete, wurden Kollegen aus Bergen eingeladen, um darin Studien über Magenmotilität mit der real time Sonografie und der berühmten Fleischsuppe des Dr. Trygve Hausken sowie über Endosonografie zu publizieren.

Für Karlheinz Seitz war Harald Lutz mit seinem Sachverstand ein zuverlässiger Partner und Ratgeber. Gemeinsam veranstalteten sie über Jahre (1996 - 2010) die hochkarätige Veranstaltung "Sonografie in der Gastroenterologie“. Dies war die erste Veranstaltung der Euroson-School der EFSUMB in Deutschland. Die Veranstaltungen waren getragen von Innovation, Kollegialität und einem besonderen Rahmenprogram mit kulturellen, sportlichen und persönlichen Begegnungen. Besonders bemerkenswert ist auch Lutz Engagement für die Zeitschrift „Ultraschall in der Medizin“ nach der Einstellung der von ihm bei Springer herausgegebenen Ultraschallzeitschrift. Sein Einsatz war stets uneigennützig und im Interesse der Sache.

\section{Noch ein paar Worte zur Person Harald} Lutz.

Zwar außerhalb Frankens geboren ist $\mathrm{Ha}$ rald Lutz dennoch Franke durch und durch: aufgewachsen in Bamberg, Studium und 
Ausbildung in Erlangen, und bis zur Pensionierung in Bayreuth tätig. Franken gelten als Charakterköpfe, sie sind - entgegen dem provinziellen Image der Region - nicht engstirnig, sondern durchaus großzügig, weltoffen und zuverlässig, wenn es darauf ankommt als belastbar und ggf. resistent bekannt.

Harald Lutz kann nicht ohne seine tüchtige und „sonophile“ Ehefrau Hedi gewürdigt werden. Wir kennen sie als humorvoll und charmant von vielen Veranstaltungen anlässlich vieler Dreiländertreffen.

Wer Harald Lutz sieht, nimmt ihm die 80 Jahre nicht ab. Aufrecht, fit mit elastischem Gang und wachem, interessiertem Blick wirkt er dynamisch - ganz so als hätte er sich noch einiges vorgenommen.

Zum Geburtstag möchten wir Harald persönlich gratulieren und ihm für unsere lang- jährige Freundschaft und seinen fachlichen Rat und eine Unterstützung danken.

Den Glückwünschen schließt sich der Präsident der DEGUM PD Dr. med. Kai-Sven Heling mit dem gesamten Vorstand an.

Ad multos annos!

Karlheinz Seitz, Bernd Frentzel-Beyme, Svein Ödegaard und Josef Menzel 